\title{
Divergence of Granular Sludges and Microbial Communities in Two Types of Anaerobic Reactors Treating Different Wastewaters
}

\author{
Xianchao Qin ${ }^{1}$, Chunjie $\mathrm{Li}^{1}$, Yueshu Gao ${ }^{1}$, Zhenjia Zhang ${ }^{1 *}$, and Xiaojun Zhang ${ }^{2 *}$ \\ ${ }^{1}$ School of Environmental Science and Engineering, Shanghai Jiao Tong University, Shanghai 200240, P.R. China \\ ${ }^{2}$ State Key Laboratory of Microbial Metabolism, and Joint International Research Laboratory of Metabolic and Developmental Sciences, and \\ School of Life Sciences and Biotechnology, Shanghai Jiao Tong University, Shanghai 200240, P.R. China
}

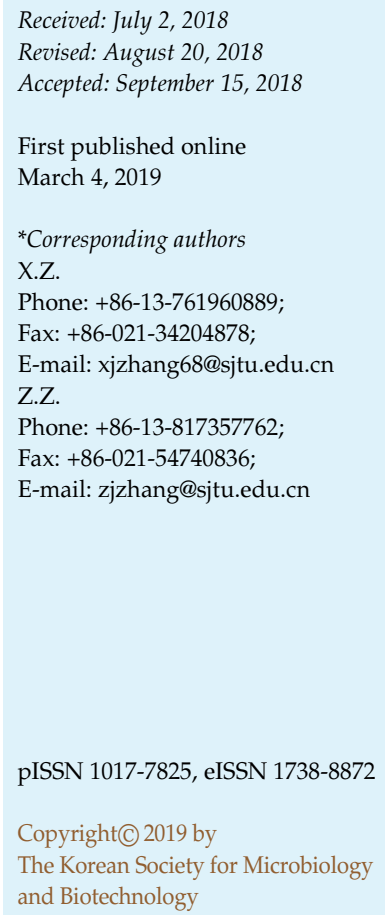

An advanced anaerobic expanded granular sludge bed (AnaEG) and an internal circulation (IC) reactor, which were adopted to treat starch processing wastewater (SPW) and ethanol processing wastewater (EPW), were comprehensively analyzed to determine the key factors that affected the granules and microbial communities in the bioreactors. The granule size of $900 \mu \mathrm{m}$ in the AnaEG reactor was smaller than that in the IC reactor, and the internal and external morphological structures of the granular sludge were also significantly different between the two types of reactors. The biodiversity, which was higher in the AnaEG reactor, was mainly affected by reactor type. However, the specific microbial community structure was determined by the type of wastewater. Furthermore, the dominant methanogens of EPW were mainly Methanosaeta and Methanobacterium, but only Methanosaeta was a major constituent in SPW. Compared with the IC reactor, characteristics common to the AnaEG reactor were smaller granules, higher biodiversity and larger proportion of unknown species. The comparison of characteristics between these two reactors not only aids in understanding the novel AnaEG reactor type, but also elucidates the effects of reactor type and wastewater type on the microbial community and sludge structure. This information would be helpful in the application of the novel AnaEG reactor.

Keywords: Anaerobic bioreactor, microbial community, wastewater, granular sludge

\section{Introduction}

As the most prominent biotechnology for industrial wastewater treatment, anaerobic digestion is crucial for global environmental protection and the sustainable development of energy [1]. However, the general perception that anaerobic bioreactors are unreliable or unstable, owing to the undefined microbial composition underlying the different anaerobic reactors or wastewaters, inhibits their wider application. Syntrophic assemblages of microorganisms, such as hydrolytic, acidogenic and acetogenic bacteria, and methanogenic archaea, play important roles in the diverse anaerobic pathways of substrate metabolism [2]. Therefore, a thorough comparative investigation of the microbial community structures may reveal the nature of anaerobic bioreactors processing different wastewaters, and this may facilitate their wider use and development.

Microorganisms are the primary interest because they perform the anaerobic digestion. The predominant microorganisms in anaerobic bioreactors have been revealed to a large extent by previous studies [3,4]. Some researchers have also attempted to compare the microbial community structures of several kinds of wastewater to determine differences in their microbial distribution patterns. Narihiro et al. quantitatively monitored the trophic groups of bacteria in methanogenic communities of six different anaerobic processes using the sequence-specific rRNA cleavage method, successfully targeting the predominant semisyntrophic bacteria, Chloroflexi, and propionate-oxidizing syntrophs including Syntrophobacter, Smithella and Pelotomaculum [5]. 
Comparative studies of several full-scale anaerobic reactors have also been conducted to summarize the characteristics shared by similar anaerobic reactors or wastewater. For instance, 125 full-scale anaerobic treatment plants were investigated to provide insight into handling high nitrate concentrations and preventing the emergence of lime, magnesium ammonium phosphate or aluminum precipitation [6]. Microbial community composition and structure have also been determined in comparative studies. For example, investigation of nine full-scale bioreactor facilities for brewery wastewater treatment ultimately determined a unique community structure with unprecedented stability [7]. The uniqueness of microbial communities in different anaerobic facilities was further affirmed by a later study, in which it was determined that Firmicutes were the predominant population in four fullscale anaerobic digestion plants, although each plant hosted a unique microbial community [8]. The above studies investigated bacterial community structure in the same type of wastewater, but each reactor had its own set of specific characteristics; therefore, it remains worthwhile to consider the universality of the above results. Hence, considering the shortcomings of the above research, and in order to better understand the factors influencing the formation of a specific microbial community structure, it is necessary to study the microbial community characteristics when the same reactor is used to treat different wastewaters and when the same wastewater is treated by different reactors.

Among the numerous anaerobic bioreactors, the expanded granular sludge bed (EGSB) reactor has attracted more attention in recent years due to its improved performance and economic efficiency during the anaerobic digestion of municipal wastewater $[9,10]$. However, unstable performance and a long startup period are still challenging problems for the EGSB reactor [11]. Accordingly, an advanced EGSB (AnaEG) reactor was developed by Zhang [12]. The lack of effluent circulation is its most important merit with regard to energy conservation, and the plug flow pattern offers a well-dispersed upward influent flow to guarantee the full expansion of the granular sludge. The AnaEG has been successfully applied to treat a variety of industrial wastewaters, such as coal gasification wastewater [12], starch processing wastewater (SPW) and ethanol processing wastewater (EPW).

Our previous research [13] showed that the AnaEG reactor maintained excellent and stable performance for SPW treatment, even with fluctuating conditions in influent wastewater quality. Temporal stability and spatial homogeneity typified the microbial community of the AnaEG reactor treating SPW. These results were highly consistent with the temporal and spatial treatment performance. In contrast, a stratified distribution of the microbial community is typically observed in other studies $[14,15]$. Hence, spatial homogeneity in the distribution of microbes seems to be a special feature of the AnaEG reactor. The excellent performance of the AnaEG reactor in the treatment of SPW and its distinct spatial distribution of microorganisms prompted us to ask whether this microbial distribution would occur during treatment of other types of wastewater. There is also a type of wastewater treatment plant (WWTP) that has adopted the AnaEG and the internal circulation (IC) reactors to treat EPW in parallel. An AnaEG reactor at a WWTP treating SPW and AnaEG and IC reactors at a WWTP treating EPW were considered to be excellent systems for comparing and understanding the microbial mechanism of different anaerobic reactors.

In this study, we had several objectives under the aim of better understanding the microbial characteristics of different wastewaters and bioreactors to guide the management of anaerobic bioreactors. The major objectives were $(i)$ to determine if there were specific granular and microbial characteristics of identical reactors applied to treat different wastewaters, (ii) to determine the microbial community structures of different bioreactors used to treat the same wastewater, and (iii) to identify the decisive factor in the formation of microbial community structures that differ between wastewaters and reactors. To solve the above issues, we employed morphological analyses to observe differences in the anaerobic granular sludge (AGS) and Illumina MiSeq sequencing of bacterial 16S rRNA genes to analyze the microbial community structures of three fullscale anaerobic bioreactors.

\section{Materials and Methods}

\section{Wastewater and Anaerobic Reactors}

Three bioreactors within two WWTPs that were separately used for the treatment of SPW and EPW were studied. The first type of AGS was collected from a WWTP in Hangzhou, China, that treated SPW using the AnaEG reactor. AGS was also sampled from a WWTP treating EPW in Jilin, China, where an IC reactor and an AnaEG reactor were employed separately for EPW treatment. All the reactors were operated at mesophilic temperatures of $31-35^{\circ} \mathrm{C}$.

\section{Sample Collection}

Granular sludge samples taken from the bottom and upper sections of the AnaEG reactor that was treating SPW were named AnS_b and AnS_u, respectively. In the case of EPW treatment, the 
granular sludge sample taken from the bottom area of the AnaEG reactor was named AnE_b, and samples collected from the bottom and upper sections of the IC reactor were named ICE_b and ICE_u, respectively. In the above abbreviations, ' $\mathrm{An}^{\prime}$ and ' $\mathrm{IC}^{\prime}$ signify the AnaEG and IC reactors, respectively, ' $S$ ' and ' $E$ ' signify SPW and EPW, respectively, and ' $b$ ' and ' $u$ ' signify the bottom and upper sections of the reactors, respectively. All the sludge samples were stored at $4^{\circ} \mathrm{C}$ before analysis or were frozen for long term storage.

\section{Granule Size and Scanning Electron Microscope Analysis}

To evaluate the granule size distribution of the AGS, the diameters of the granules were assessed using a laser particle size analyzer (Mastersizer 3000, UK). A scanning electron microscope (SEM) was employed to observe the morphology of the AGS. After overnight pretreatment with $2.5 \%$ glutaraldehyde, the samples were dehydrated in a gradient series of ethanol solutions. Next, a liquid carbon dioxide critical evaporator (Quorum, UK) was used for sample drying, and metal spraying was conducted using an ion sputtering apparatus (Leica, Germany). Finally, an SEM (Hitachi, Japan) was employed to observe the cellular morphology of the AGS.

\section{Construction of the Sequencing Library}

Genomic DNA was extracted from AGS according to a previous method with minor modifications [16]. Two-step PCR targeting the V3-V4 regions of the 16S rRNA gene was carried out for the construction of the sequencing library. The primers 341_F and 805_R were used for PCR amplification [17]; the PCR amplifications and subsequent purification steps were conducted according to the manufacturer's (Illumina Inc., USA) protocol, described at https://support.illumina.com/content/dam/illuminasupport/documents/documentation/chemistry_documentation/ 16s/16s-metagenomic-library-prep-guide-15044223-b.pdf. Finally, the purified PCR products were analyzed by the Illumina MiSeq System (Illumina Inc.).

\section{Bioinformatics Analysis of Microbial Communities}

The preprocessing of sequencing data was conducted according to the methods described previously [18]. The Uparse algorithm with default settings was used to select the representative operational taxonomy units (OTUs) [19], and reference-based chimeric sequence detection was conducted in Uchime [20] with the RDP classifier training database [21] to generate the OTU table. Next, alpha diversity, including the Shannon index, Simpson, Chao1, observed species and Good's coverage, were assessed using the QIIME platform [22]. Principal coordinates analysis (PCoA) of the overall microbial community structure, based on weighted UniFrac distance matrices, was performed using GraphPad Prism (USA), and the unweighted pair-group method with arithmetic means (UPGMA) was used to evaluate the phylogenetic relationships between different sludge samples. Venn diagram analysis of OTUs was implemented to demonstrate the specific characteristics of different microbial communities. Finally, taxonomic classifications at the phylum and genus levels were generated by the online RDP classifier tool (http:// rdp.cme.msu.edu/classifier/classifier.jsp) with threshold bootstrap values at $80 \%$.

\section{Sequence Data Accession Numbers}

The 16S rRNA gene sequences generated in this study were submitted to the GenBank Sequence Read Archive database under the accession number SRP150070.

\section{Results}

\section{Morphological Differences in Anaerobic Granular Sludge}

Analysis of the granule size distribution of sludge from different reactors showed that the type of wastewater was the major factor affecting the difference in granule size (Fig. 1A). Granules from the reactor treating SPW (AnS_b and AnS_u) were smaller than those from the reactors treating EPW (AnE_b and ICE_b). In the reactor treating SPW, midsize granules with diameters between 330 and $1,040 \mu \mathrm{m}$ comprised more than $70 \%$ of the sludge; the proportions of the sludge comprising small $(0-330 \mu \mathrm{m})$ and large $(1,040-3,280 \mu \mathrm{m})$ granules were less than $10 \%$ and $20 \%$, respectively. In the reactors treating EPW, the sludge was mainly large granules (approximately 50\%), and the small granules comprised more than $20 \%$. Furthermore, granules from the AnaEG reactor (AnE_b) were smaller than those from the IC reactor (ICE_b), although both reactors were used to treat EPW. The average granule diameters in different reactors and wastewaters were consistent with the above results; the average granule diameter in the AnaEG reactor treating SPW was approximately $900 \mu \mathrm{m}$, but the average was approximately $1,200 \mu \mathrm{m}$ in the IC reactor treating EPW (Fig. 1B). In other words, the AnaEG reactor treating SPW formed smaller granules than the IC reactor treating EPW.

As observed by SEM, there were distinct differences in the surface appearance and the inner microstructure of AGS in different wastewaters and reactors (Fig. 2). In the AnaEG reactor treating SPW, the AGS consisted of small and smooth particles. Filamentous- and bacillus-shaped microorganisms predominated near the surface in SPW (surface, AnS), whereas the bacillus-shaped microorganisms intertwined randomly throughout the cross-section (cut plane, AnS). Moreover, a small bulge was observed on the surface of the AGS, and numerous bacillus- and filamentous-shaped bacteria constituted the surface matrix (surface, AnE). In the AnaEG reactor employed for EPW treatment, cocci-shaped microorganisms of many different 

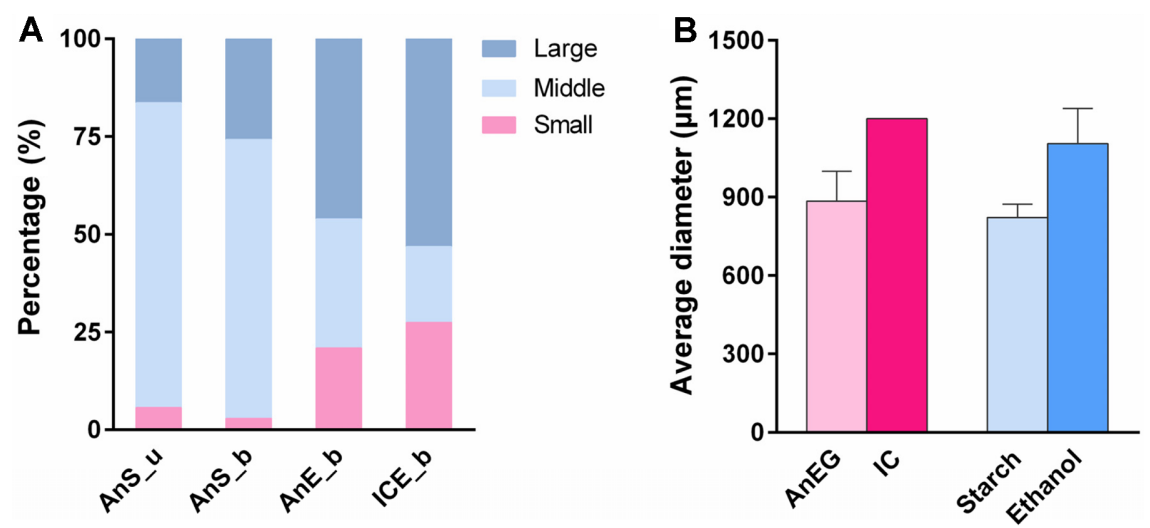

Fig. 1. Differences in granule size.

(A) The size distribution of granules, with small, middle and large granules having diameters of $0-330,330-1,040$, and 1,040-3,280 $\mu$ m, respectively. (B) The average diameter of sludge granules in different reactors and wastewater.

sizes were widely distributed throughout the cross-section (cut plane, AnE). Furthermore, although both AnaEG and IC reactors were used to treat EPW, the granule morphology and microbial composition in the two reactors were quite different. AGS in the IC reactor consisted of large elliptical particles with uneven surfaces, but no obvious bulge was observed. Bacillus- and filamentous-shaped microorganisms were located on the surface of the AGS (surface, ICE). In the inner layer, there was an observable niche formed by bacillus-shaped microorganisms surrounded by many filamentous-shaped microorganisms (cut plane, ICE). The results suggested that the type of wastewater affected the appearance and morphology of the AGS, but the type of reactor was also an important factor, even for the same wastewater.

\section{Species Diversity in Different Reactors}

The alpha diversity indices are displayed in Table 1 and Fig. 3. The Good's coverage of all samples was above 0.996, indicating that the current sequencing depth accurately

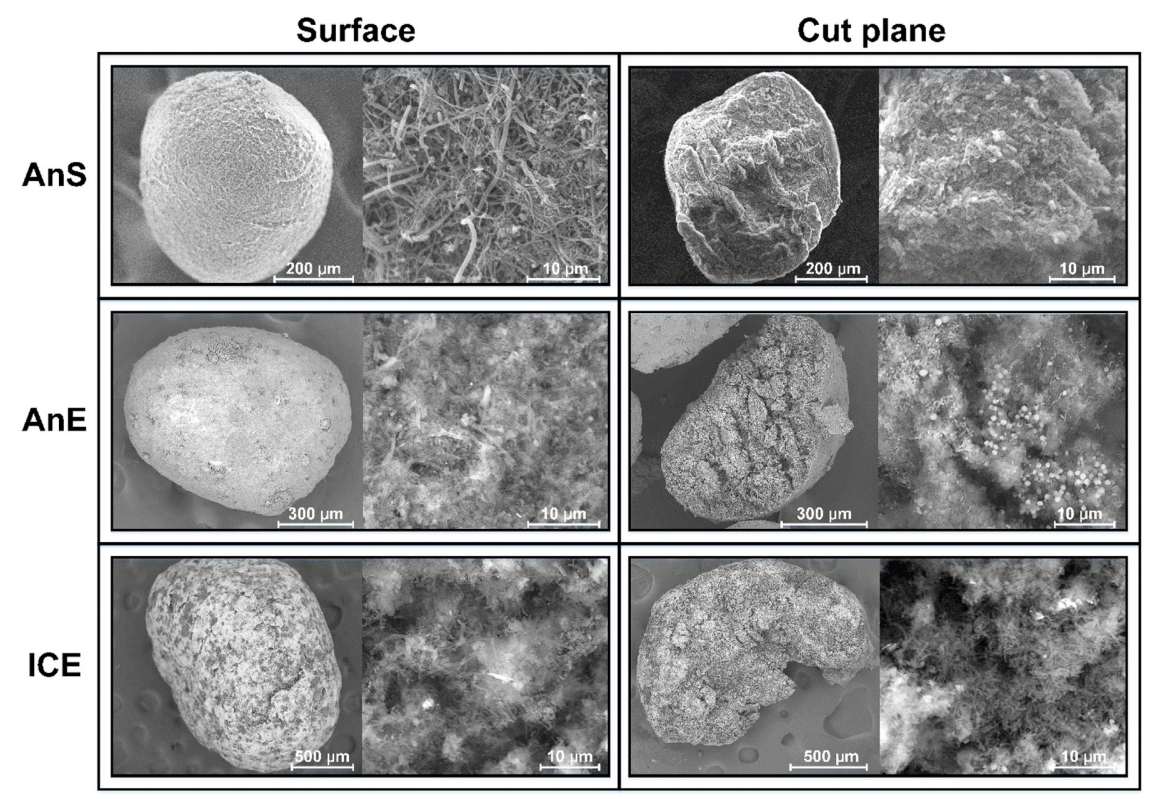

Fig. 2. SEM images of granular sludge from different reactors.

AnS and AnE represent sludge sampled from the AnaEG reactors treating SPW and EPW, respectively; ICE represents a sludge sampled from the IC reactor treating EPW. The surface and inner layers are shown in the left and right columns, respectively. 
Table 1. Alpha diversity index of the different AGS samples.

\begin{tabular}{ccccccc}
\hline Wastewater & Sample ID & Shannon index & Chao1 & Simpson & Observed species & Good's coverage \\
\hline SPW & AnS_b & 6.28 & 605.61 & 0.97 & 558 & 0.996 \\
& AnS_u & 6.18 & 579.43 & 0.97 & 525 & 0.997 \\
EPW & AnE & 6.12 & 607.96 & 0.97 & 532 & 0.997 \\
& ICE_b & 5.83 & 585.71 & 0.96 & 475 & 0.996 \\
& ICE_u & 5.5 & 544.15 & 0.93 & 443 & 0.996 \\
\hline
\end{tabular}

reflected the microbial community in the samples. The diversity of the microbial community differed between the two types of reactors, but no significant difference was detected between the two types of wastewater. The Shannon index of the AnaEG reactor was 6.19, which was significantly higher than that of the IC reactor (5.67), even when processing the same wastewater (EPW) (data not shown). However, no difference was observed between the SPW and EPW (Fig. 3A). The number of species observed in the AnaEG reactor was 538, but only 459 were observed in the IC reactor, which agreed with the results of the Shannon index (Fig. 3D). However, no significant differences were observed in the Chao1 (Fig. 3B) and Simpson (Fig. 3C) indices. These results indicated that the AnaEG reactor possessed a higher number of species and greater species diversity compared to the IC reactor.

\section{Distinctive Overall Microbial Community Structures in Different Reactors}

PCoA, based on an unweighted UniFrac distance matrix, detected similar microbial community structures in the lower and upper sections of the same reactor, and different reactors and wastewaters shaped the specific microbial community structure (Fig. 4A). In general, PC1 contributed $80.9 \%$ of the interpretation of the overall microbial distribution, and samples from different wastewaters were
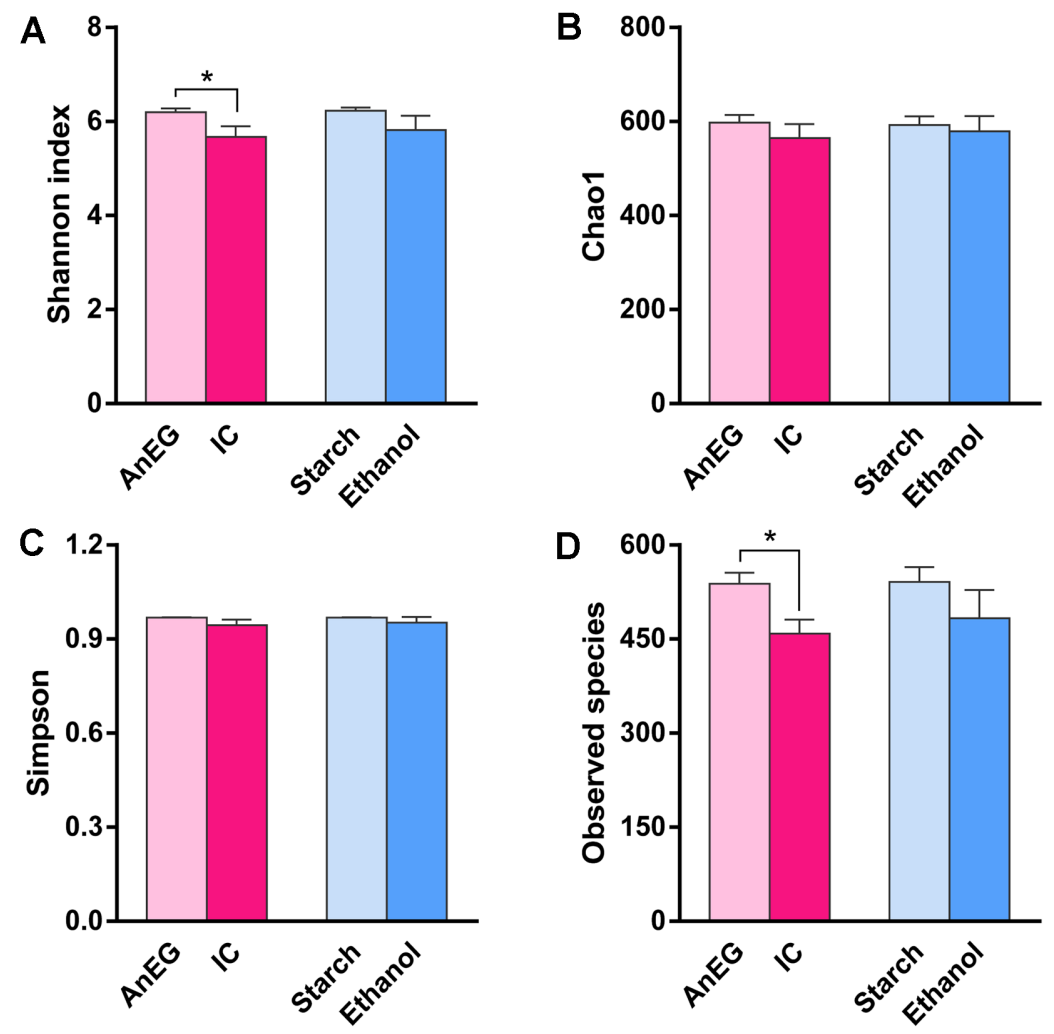

Fig. 3. Alpha diversity of anaerobic granular sludges.

The indices include (A) the Shannon index, (B) Chao 1, (C) Simpson, and (D) observed species. ${ }^{*} p<0.05$ using paired t-test. 

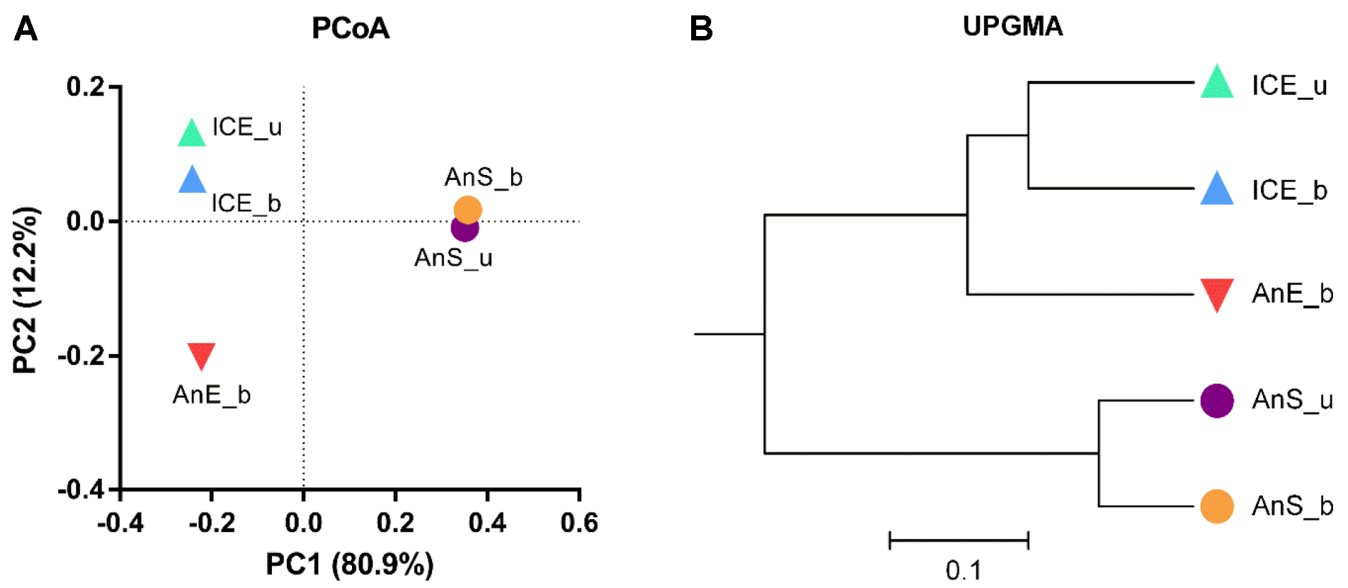

Fig. 4. Differences in overall microbial community structure.

(A) PCoA, based on the unweighted UniFrac distance, of the microbial community structures of sludge sampled from different reactors and wastewaters. (B) Phylogenetic tree based on the unweighted pair-group method with arithmetic means (UPGMA).

grouped far apart along the PC1 axis. Hence, substrate differences were the major reason for the formation of a specific microbial community structure. In addition, samples from different reactors were separated from each other along the PC2 axis (contributing $12.2 \%$ to the total variation); hence, the type of reactor also affected the microbial composition. The UPGMA tree further confirmed that the largest genetic distances existed between samples from different wastewaters, but more similar phylogenetic relationships were present in the same reactor type, even when it was used to treat different wastewaters (Fig. 4B). Therefore, both wastewater and reactor type could affect the microbial community structure, but the former was the major determinant.

Venn diagram analysis was carried out to further dissect the microbial composition differences between samples from different reactors and wastewaters (Fig. 5A). Of 1,035 OTUs, 110 were shared members that accounted for $50.66 \%$ relative abundance of the total OTUs, although the percentage of shared OTUs only accounted for $10.63 \%$ of the total OTUs. These results illustrated that there were many common microorganisms in the two kinds of wastewaters and that the OTUs were concentrated in a few microbial populations. AnE_b possessed 77 unique OTUs, followed by AnS_b (26) and ICE_b (20), whereas AnS_u (16) and ICE_u (7) contained the fewest unique OTUs. Moreover, the percentage and relative abundance of unique OTUs were highest in AnE_b, and this bottom region contained more unique OTUs than the upper region of the same reactor (Fig. 5B). This implies that the AnaEG reactor supported more specific microbes, of which Proteobacteria
(19.6\%), Chloroflexi $(9.4 \%)$ and Euryarchaeota (8.7\%) constituted the most predominant phyla among the shared OTUs. Moreover, many unclassified microorganisms (9.4\%) existed in these samples (Fig. 5C). At the genus level (Fig. 5D), Geobacter (13.0\%), which can couple ethanol, acetate, formate or lactate oxidation with the reduction of iron or manganese oxides [23], was the most predominant genus among the shared OTUs. Syntrophobacter (2.4\%) and Syntrophorhabdus $(1.3 \%)$ also showed high relative abundances. The former has the ability to degrade propionate and fumarate [24] and has been thought to be a species that can degrade toxic organic matter, such as quinoline [17]. Syntrophorhabdus is a potential syntrophic phenol-degrading bacterium [25]. Some methanogens, such as Methanosaeta (5.4\%) and Methanobacterium (3.1\%), which are acetoclastic [26] and hydrogenotrophic methanogens [27], respectively, also constituted high proportions of the shared OTUs.

\section{Comparison of Predominant Bacteria in Different Reactors}

The taxonomic composition of predominant microbial populations revealed that different reactors and wastewaters supported correspondingly different functional microbial communities. In the SPW and EPW samples, $62.9 \%$ and $83.9 \%$ of the sequences, respectively, were identified at the phylum level (Fig. 6A). The most predominant member in samples from EPW was Proteobacteria, with an average relative abundance of $35 \%$. Its relative abundance was higher in the upper region of the IC reactor (ICE_u $=42.6 \%$ ) than in the bottom region (ICE_b $=30.7 \%$ ). However, this phylum constituted only $13.5 \%$ in samples of SPW, and no obvious difference was detected between the bottom and 
A

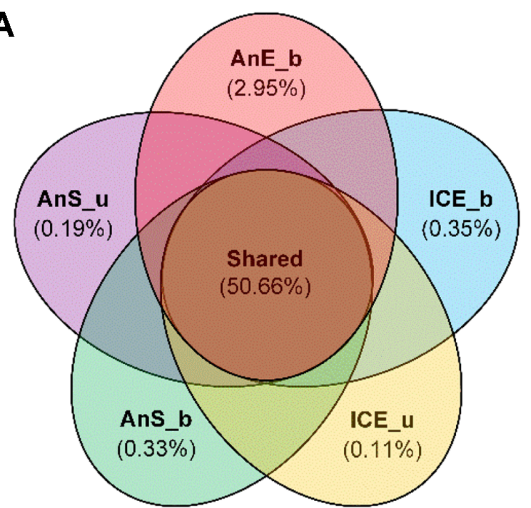

Total OTU=1035

C

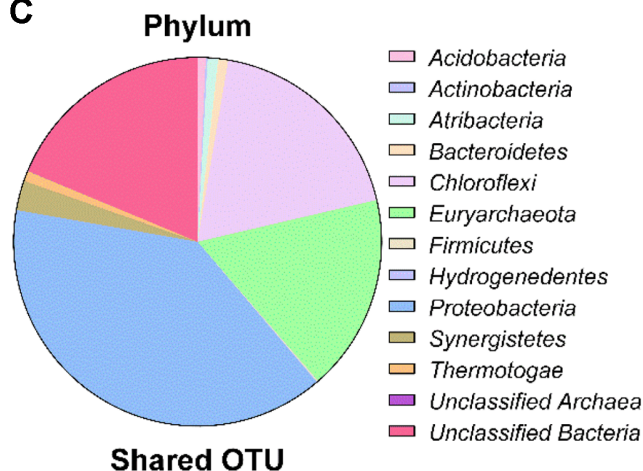

B
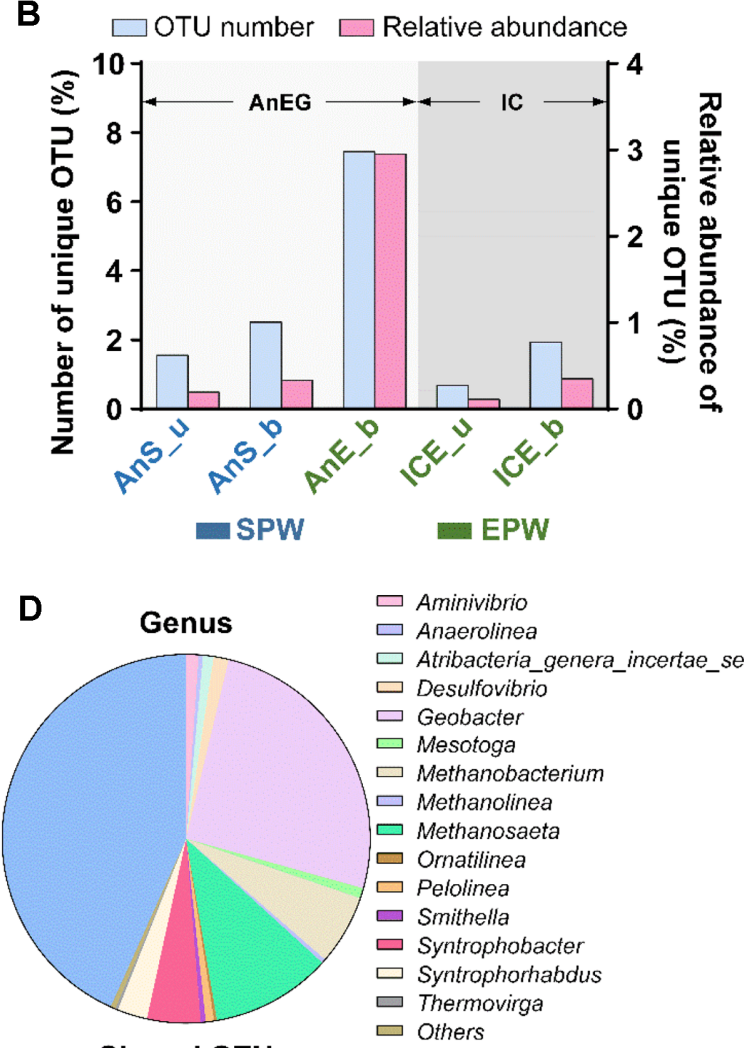

$\square$ Anaerolinea

$\square$ Atribacteria_genera_incertae_sedis

$\square$ Desulfovibrio

$\square$ Geobacter

$\square$ Mesotoga

$\square$ Methanobacterium

$\square$ Methanolinea

$\square$ Methanosaeta

$\square$ Ornatilinea

$\square$ Pelolinea

$\square$ Smithella

$\square$ Syntrophobacter

$\square$ Syntrophorhabdus

$\square$ Thermovirga

$\square$ Others

Shared OTU

Fig. 5. Microbial composition of samples from different reactors and wastewaters.

(A) Venn diagram of all OTUs, where the numbers in the brackets represent the relative abundances of the corresponding OTUs. (B) Number, percentage and relative abundance of unique OTUs. (C) and (D) present the taxonomic distribution of shared OTUs at the phylum and genus levels, respectively.

upper regions. Chloroflexi also constituted a high proportion (average $=15.5 \%$ ) in all samples, but Bacteroidetes was relatively more abundant in ICE_b (18.8\%) than in other samples (average $=5.9 \%$ ). The abundance of Firmicutes was
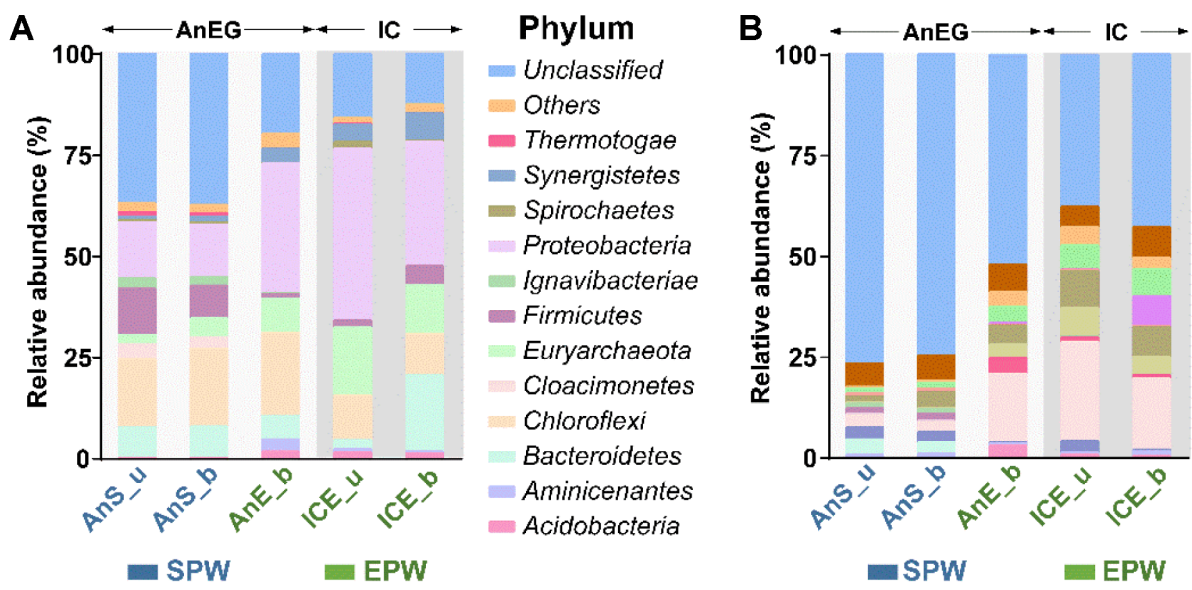

\section{Genus}

- Unclassified

- Others

- Syntrophorhabdus

- Syntrophobacter

- Prevotella

- Pelolinea

- Methanosaeta

Methanobacterium

- Mesotoga

- Mangrovibacterium

- Longilinea

- Geobacter

- Desulfovibrio

- Candidatus Cloacamonas

- Aminivibrio

- Aminicenantes_genera

incertae sedis

Fig. 6. The taxonomic distribution of the microbial communities.

(A) Phylum level. (B) Genus level. 
higher in the SPW samples (average $=9.7 \%$ ) than in the EPW samples (average $=2.4 \%$ ). Other phyla, including Cloacimonetes, Ignavibacteriae, and Thermotogae, were dominant microbial community members in SPW samples, but they were rare members in EPW samples. In contrast, bacteria belonging to the phyla Acidobacteria, Aminicenantes, Synergistetes and Spirochaetes were the dominant populations in the EPW samples but were only detected at low abundances in the SPW samples. Additionally, more Euryarchaeota were detected in EPW samples (average $=12.4 \%$ ) than in samples from SPW (average $=3.5 \%$ ).

At the genus level (Fig. 6B), Geobacter was the most predominant member in EPW samples, with an average relative abundance of $19.7 \%$, but only comprised $3 \%$ of the population in SPW samples. Syntrophobacter (average $=5.5 \%$ ) and Syntrophorhabdus (average $=3.7 \%$ ) were also more abundant in the EPW samples than in SPW. Therefore, although the Venn diagram indicates that these three genera were of high relative abundance among the shared species, they were mainly present in the EPW samples. Notably, Longilinea (average $=2.0 \%$ ), a filamentous bacterium of the phylum Chloroflexi [28] capable of carbohydrate and amino acid degradation [29], was the predominant genus in EPW samples, but it was not detected in SPW samples. In addition, the hemicellulose-degrading phylum Prevotella constituted a high proportion of the bacteria in ICE_b $(7.6 \%)$; however, it was rarely found in other samples (average $<0.5 \%$ ). Conversely, higher relative abundances of Candidatus, Cloacamonas and Desulfovibrio were observed in samples of SPW compared to EPW, and Mangrovibacterium was only detected in SPW samples (average $=1.7 \%$ ). The percentage of unclassified genera in SPW (average $=75.6 \%$ ) was higher than that in EPW (average $=44.2 \%$ ). Even in the same wastewater (EPW), the proportion of unclassified genera in the AnaEG reactor was higher than that in the IC reactor, especially in the bottom section. There are many potential functional species that may exist amid these unclassified microorganisms. These results show that samples of EPW were predominated by more classified microorganisms than what was found in other samples, but the bottom area of the bioreactor supported more species with potential functional roles in industrial wastewater treatment, especially in the AnaEG reactor.

\section{Comparison of Archaea in Different Reactors}

Methanogens constitute the majority of archaea in anaerobic bioreactors, and they play an important role in the anaerobic degradation of organic pollutants. Their abundance and composition in AGS should significantly affect the wastewater treatment efficiency. Hence, it is necessary to assess their population composition and abundance in different reactors and wastewaters. The average proportion of archaea was $12.5 \%$ in EPW samples but was only $3.5 \%$ in SPW samples. Their quantity in the upper area of the IC reactor was significantly higher than that in the bottom area (Fig. 7A). The composition of the methanogens also differed between the two reactors and wastewaters (Fig. 7B). In SPW samples, Methanosaeta (average $=2.7 \%$ ) was the predominant methanogen, and the relative abundances of Methanobacterium, Methanolinea and Methanomassiliicoccus were less than $0.5 \%$. In the EPW samples, Methanosaeta (7.1\%) and Methanobacterium (5.0\%) were relatively more abundant, while Methanolinea and Methanomassiliicoccus were not the major methane-producing archaea.
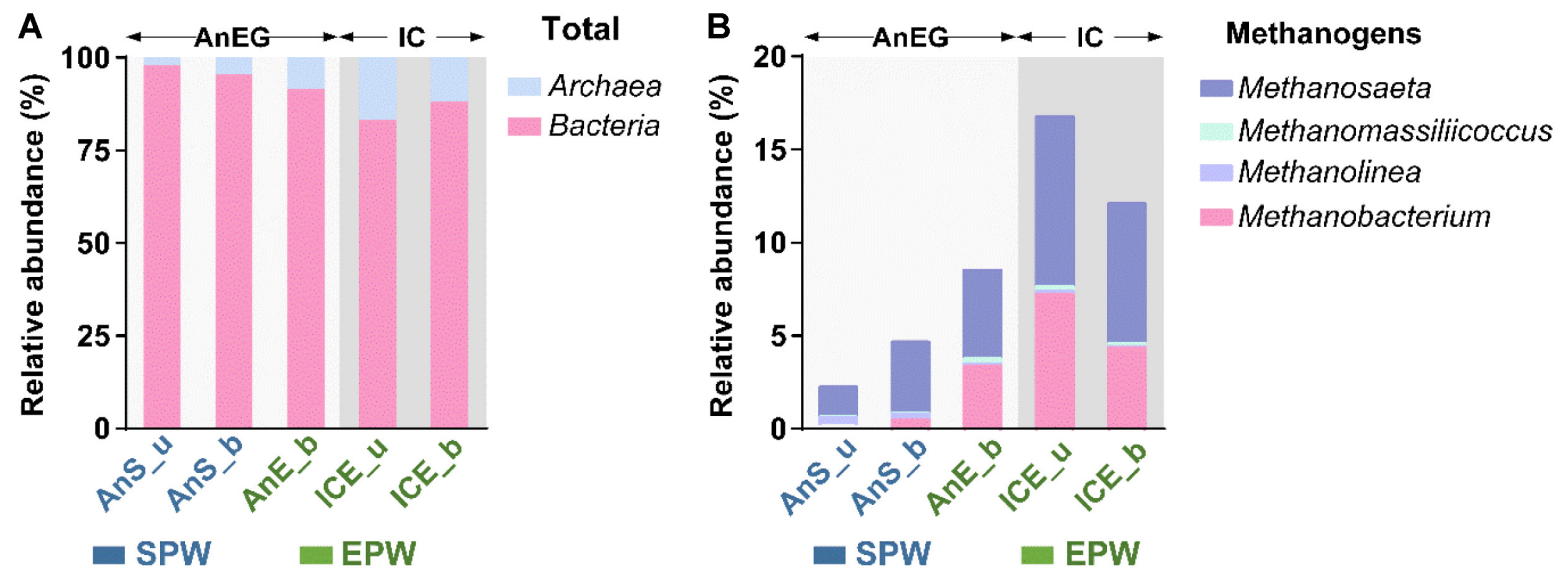

Fig. 7. The percentage of archaea in samples.

(A) Proportions of bacteria and archaea. (B) Compositions of methanogens. 


\section{Discussion}

The average granule size of AGS in the AnaEG reactors treating SPW was much smaller than that of other reported anaerobic AGS [30-32]. In this study, the comparison of granule samples from both AnaEG and IC reactors treating EPW in the same WWTP convincingly demonstrated that the granule size of AGS formed in the AnaEG was smaller. Wu et al. found that increased hydraulic shear force affects the formation of EPS and the gas-generating activity of AGS, thus increasing the granule size of AGS [33]. The internal circulation system of the IC reactor increases the upflow velocity of the wastewater and produces higher hydraulic shear force. This could account for the formation of larger granules of AGS in the IC reactor. However, unlike the traditional EGSB reactor, the AnaEG reactor has no external circulation system. The small upflow velocity favored the formation of smaller granules in the AnaEG reactor. However, larger AGS granules are disadvantageous to the anaerobic reactor. Oversized AGS causes the floating and discharge of sludge. It has been reported that smaller AGS can achieve more satisfactory wastewater treatment performance [34]. Smaller particles have larger specific surface areas, thus increasing the contact area between the wastewater and the microorganisms, which improves the mass transfer efficiency of wastewater. The cavities and cracks present on the surface of granular sludge may represent the escape route of produced biogas [31]. Alternatively, filamentous-shaped microorganisms might also act as channels for substrates and metabolites to enter and exit the granules, ensuring the transportation efficiency of the substrate and high methane productivity [35]. Consequently, although no obvious cavities and cracks were present on the surface of the AGS in this study, the numerous filamentous-shaped microorganisms may transport the metabolites and methane efficiently.

In terms of microbial composition, although the type of reactor was the main factor affecting species diversity, the microbial community structure was primarily determined by the type of wastewater. Other research has indicated that substrate differences govern the microbial composition in bioreactors [36], but some researchers claim that different bioenergy systems possess unique and resilient bacterial community structures [7]. In these studies, the reactor and wastewater communities were different from each other. The effect of too many variables may cause their results to be inconsistent with ours. Although the microbial community structure in SPW and EPW was significantly different, Venn diagram analysis showed that approximately
$50 \%$ of the bacteria in all samples were common to both wastewater types. These microorganisms were mainly composed of syntrophic bacteria (Geobacter, Syntrophorhabdus, Syntrophobacter) and methanogens (Methanosaeta and Methanobacterium). The low amounts of toxic carbohydrate pollutants in SWP and EPW enriched for phylogenetically similar microorganisms.

In addition, the number of specific microorganisms was higher in the AnaEG reactor than in the IC reactor, especially for EPW treatment. A higher proportion of methanogens was observed in EPW compared to SPW, which implies more methane production in the former, and the porous surface morphology of AGS in EPW supports this point. The probable reason for this phenomenon may lie in the differences in composition of the substrates. SPW is generated in the process of modified starch production, and its raw material is starch powder. Hence, the composition of SPW is relatively simple, and SPW is mainly composed of soluble and insoluble starch. In contrast, EPW is generated from the production of fuel ethanol, and it contains more complicated organic compounds, such as starch, sugar, cellulose, and crude protein [37]. In addition, a previous study suggested that complex substrates can form larger granules in sludge than can simple substrates [38]. Hence, the granules in EPW were larger than those in SPW. In brief, substrate may be an important factor affecting the relative abundance of some bacteria.

According to our previous study [13], the AnaEG reactor used for SPW treatment did not show differences in vertical microbial distribution, and it was described as a homogeneous system. In contrast, the composition of both the overall microbial community and the methanogens was obviously different between the bottom and upper areas of the IC reactor. This result indicates that different organic degradation reactions may occur in the two areas, and the special design of the IC reactor may cause this phenomenon. The IC reactor consists of two reaction chambers with a tri-phase separator in the upper part of each chamber [39]; its high upflow velocity could enhance mass transfer to achieve a higher COD removal rate and methane yield [40]. However, the organic matter may not degrade completely in the bottom area of the reactor, leading to some secondary metabolism in the upper part of the reactor. Consequently, the generation of methane as the terminal reaction of the anaerobic metabolism of organic matter would be carried out in the upper part of the IC reactor, and a higher relative abundance of methanogens could be observed here. Additionally, physical isolation within the reactor was reported by Xing et al. to affect the 
spatial distribution of microbial community structure, with the authors finding methanogens, acetogens and hydrolytic fermentative bacteria in the upper, middle and lower chambers, respectively, of the CAR reactor [15].

One of the dominant bacteria in the IC reactor, belonging to the genus Prevotella, has been reported as a hemicellulosedegrading bacterium [41]. It implied that these microorganisms utilize polysaccharide substrates. However, at the top of the IC reactor, these microorganisms were not dominant bacteria, probably due to the depletion of substrates. Another hydrolytic bacterial genus, Longilinea, was dominant only in the AnaEG reactor treating EPW. According to the literature, when cocultured with hydrogenutilizing methanogens, the growth rate of Longilinea is remarkably increased [28]. This was the case in our study, where a high proportion of hydrogen-utilizing methanogens (e.g., Methanobacterium) was present in the same site with Longilinea due to the spatial homogeneity inside the AnaEG reactor. However, because the substrates for Longilinea and hydrogen-utilizing methanogens were separated in two isolated spaces in the IC reactor, Longilinea could not meet the required growth condition. Therefore, the abundance of Longilinea in the IC reactor was much less than that in the AnaEG reactor.

Methanosaeta and Methanobacterium are acetoclastic [26] and hydrogenotrophic methanogens [27], respectively. The acetoclastic pathway may be the major metabolic pathway of methane synthesis in SPW anaerobic degradation, but both acetoclastic and hydrogenotrophic methanogenesis simultaneously mediated methane production during anaerobic degradation of EPW. On the other hand, more methanogens were detected at the bottom of the AnaEG reactor treating SPW than the top, but the opposite was observed in the IC reactor treating EPW. This result implies that different degradation processes may exist in these two reactors. Moreover, the special structural design of the reactor can shape the vertical distribution of the microbial community structure. The components within the wastewater are the main factor that causes differences in microbial community structure; meanwhile, the type of reactor also affects the species diversity.

In conclusion, common characteristics of the AnaEG reactor include small granular sludge, a higher abundance of unknown species, and higher biodiversity; these characteristics distinguish the AnaEG reactor from other reactors. The type of reactor is the main factor affecting biodiversity, but the composition of the wastewater is the key factor shaping the specific microbial community structure.

\section{Acknowledgments}

This study was funded by the National Natural Science Foundation of China (NSFC 31670105, 21177086). The authors would like to express their gratitude to Salma Tabassum for her English editing. The authors also would like to thank technicians at Hangzhou STARPRO Starch Mill and Jilin Fuel Ethanol Co., Ltd., China, for their assistance in sampling.

\section{Conflict of Interest}

The authors have no financial conflicts of interest to declare.

\section{References}

1. Angenent LT, Karim K, Al-Dahhan MH, Wrenn BA, Domiguez-Espinosa R. 2004. Production of bioenergy and biochemicals from industrial and agricultural wastewater. Trends Biotechnol. 22: 477-485.

2. Aydin S, Ince B, Ince O. 2015. Application of real-time PCR to determination of combined effect of antibiotics on Bacteria, Methanogenic Archaea, Archaea in anaerobic sequencing batch reactors. Water Res. 76: 88-98.

3. Ambuchi JJ, Liu JF, Wang HM, Shan LL, Zhou XT, Mohammed MOA, et al. 2016. Microbial community structural analysis of an expanded granular sludge bed (EGSB) reactor for beet sugar industrial wastewater (BSIW) treatment. Appl. Microbiol. Biotechnol. 100: 4651-4661.

4. Wang DP, Liu B, Ding XC, Sun XB, Liang Z, Sheng SX, et al. 2017. Performance evaluation and microbial community analysis of the function and fate of ammonia in a sulfatereducing EGSB reactor. Appl. Microbiol. Biotechnol. 101: 77297739.

5. Narihiro T, Terada T, Ohashi A, Kamagata Y, Nakamura K, Sekiguchi Y. 2012. Quantitative detection of previously characterized syntrophic bacteria in anaerobic wastewater treatment systems by sequence-specific rRNA cleavage method. Water Res. 46: 2167-2175.

6. Austermann-Haun U, Meyer H, Seyfried CF, Rosenwinkel KH. 1999. Full scale experiences with anaerobic/aerobic treatment plants in the food and beverage industry. Water Sci. Technol. 40: 305-312.

7. Jeffrey JW, Dan K, Marcelo LG, Nicholas BS, Samual S, Kevin Y, et al. 2011. Bacterial community structures are unique and resilient in full-scale bioenergy systems. PNAS 108: 4158-4163.

8. De Vrieze J, Raport L, Roume H, Vilchez-Vargas R, Jauregui R, Pieper DH, et al. 2016. The full-scale anaerobic digestion microbiome is represented by specific marker populations. Water Res. 104: 101-110. 
9. Fang C, Boe K, Angelidaki I. 2011. Biogas production from potato-juice, a by-product from potato-starch processing, in upflow anaerobic sludge blanket (UASB) and expanded granular sludge bed (EGSB) reactors. Bioresour. Technol. 102: 5734-5741.

10. Fettig J, Pick V, Austermann-Haun U, Blumberg M, Phuoc NV. 2013. Treatment of tapioca starch wastewater by a novel combination of physical and biological processes. Water Sci. Technol. 68: 1264-1270.

11. Zhao LK, Li XM, Mo CR, Zhang CL, Cui W, Xie T. 2012. Effect of Low concentration cyanide on startup of Expanded Granular Sludge Bed for the treatment of tapioca starch wastewater. Adv. Environ. Sci. Eng. 518-523: 2493-2499.

12. Li CJ, Tabassum S, Zhang ZJ. 2014. An advanced anaerobic expanded granular sludge bed (AnaEG) for the treatment of coal gasification wastewater. Rsc Adv. 4: 57580-57586.

13. Qin XC, Wu XG, Li LF, Li CJ, Zhang ZJ, Zhang XJ. 2018. The advanced anaerobic expanded granular sludge bed (AnaEG) possessed temporally and spatially stable treatment performance and microbial community in treating starch processing wastewater. Front. Microbiol. 9: 589.

14. Antwi P, Li JZ, Boadi PO, Meng J, Shi E, Xue C, et al. 2017. Functional bacterial and archaeal diversity revealed by $16 \mathrm{~S}$ rRNA gene pyrosequencing during potato starch processing wastewater treatment in an UASB. Bioresour. Technol. 235: 348-357.

15. Xing YJ, Ji JY, Zheng P, Zhang JQ, Ghulam A. 2014. Microbial consortium and its spatial distribution in a compartmentalized anaerobic reactor. Appl. Microbiol. Biotechnol. 98: 1357-1366.

16. Griffiths RI, Whiteley AS, O'Donnell AG, Bailey MJ. 2000. Rapid method for coextraction of DNA and RNA from natural environments for analysis of ribosomal DNA- and rRNA-based microbial community composition. Appl. Environ. Microbiol. 66: 5488-5491.

17. Wang Y, Tian H, Huang F, Long WM, Zhang QP, Wang J, et al. 2017. Time-resolved analysis of a denitrifying bacterial community revealed a core microbiome responsible for the anaerobic degradation of quinoline. Sci. Rep. 7: 14778.

18. Zhang QP, Wu YQ, Wang J, Wu GJ, Long WM, Xue ZS, et al. 2016. Accelerated dysbiosis of gut microbiota during aggravation of DSS-induced colitis by a butyrate-producing bacterium. Sci. Rep. 6: 27572.

19. Edgar RC. 2013. UPARSE: highly accurate OTU sequences from microbial amplicon reads. Nat. Methods 10: 996-1000.

20. Edgar RC, Haas BJ, Clemente JC, Quince C, Knight R. 2011. UCHIME improves sensitivity and speed of chimera detection. Bioinformatics 27: 2194-2200.

21. Cole JR, Wang Q, Fish JA, Chai B, McGarrell DM, Sun Y, et al. 2014. Ribosomal Database Project: data and tools for high throughput rRNA analysis. Nucleic Acids Res. 42: 633-642.

22. Caporaso JG, Kuczynski J, Stombaugh J, Bittinger K, Bushman FD, Costello EK, et al. 2010. QIIME allows analysis of high-throughput community sequencing data. Nat. Methods 7: 335-336.

23. Torres K, Alvarez-Hornos FJ, San-Valero P, Gabaldon C, Marzal P. 2018. Granulation and microbial community dynamics in the chitosan-supplemented anaerobic treatment of wastewater polluted with organic solvents. Water Res. 130: 376-387.

24. Popp D, Plugge CM, Kleinsteuber S, Harms H, Strauber H. 2017. Inhibitory effect of coumarin on syntrophic fatty acidoxidizing and methanogenic cultures and biogas reactor microbiomes. Appl. Environ. Microbiol. 83: 1-14.

25. Chen CL, Wu JH, Tseng IC, Liang TM, Liu WT. 2009. Characterization of active microbes in a full-scale anaerobic fluidized bed reactor treating phenolic wastewater. Microbes Environ. 24: 144-153.

26. Connelly S, Shin SG, Dillon RJ, Ijaz UZ, Quince C, Sloan WT, et al. 2017. Bioreactor scalability: laboratory-scale bioreactor design influences performance, ecology, and community physiology in expanded granular sludge bed bioreactors. Front. Microbiol. 8: 664.

27. Hao L, Bize A, Conteau D, Chapleur O, Courtois S, Kroff P, et al. 2016. New insights into the key microbial phylotypes of anaerobic sludge digesters under different operational conditions. Water Res. 102: 158-169.

28. Yamada T, Imachi H, Ohashi A, Harada H, Hanada S, Kamagata $Y$, et al. 2007. Bellilinea caldifistulae gen. nov., sp. nov. and Longilinea arvoryzae gen. nov., sp. nov., strictly anaerobic, filamentous bacteria of the phylum Chloroflexi isolated from methanogenic propionate-degrading consortia. Int. J. Syst. Evol. Microbiol. 57: 2299-2306.

29. Yan ZS, Jiang HL, Cai HY, Zhou YL, Krumholz LR. 2015. Complex interactions between the macrophyte acorus calamus and microbial fuel cells during pyrene and benzo[ $\alpha]$ pyrene degradation in sediments. Sci. Rep. 5: 10709.

30. Lu XQ, Zhen GY, Estrada AL, Chen M, Ni JL, Hojo T, et al. 2015. Operation performance and granule characterization of upflow anaerobic sludge blanket (UASB) reactor treating wastewater with starch as the sole carbon source. Bioresour. Technol. 180: 264-273.

31. Antwi P, Li J, Boadi PO, Meng J, Koblah Quashie F, Wang X, et al. 2017. Efficiency of an upflow anaerobic sludge blanket reactor treating potato starch processing wastewater and related process kinetics, functional microbial community and sludge morphology. Bioresour. Technol. 239: 105-116.

32. Sun YJ, Xing W, Li JP, Lu YQ, Zuo JE. 2009. Microbial community in granules from a high-rate EGSB reactor. Prikl. Biokhim. Mikrobiol. 45: 659-663.

33. Wu J, Bi L, Zhang JB, Poncin S, Cao ZP, Li HZ. 2012. Effects of increase modes of shear force on granule disruption in upflow anaerobic reactors. Water Res. 46: 3189-3196.

34. Wang L, Zheng $\mathrm{P}$, Xing YJ, Li W, Yang J, Abbas G, et al. 2014. Effect of particle size on the performance of autotrophic nitrogen removal in the granular sludge bed 
reactor and microbiological mechanisms. Bioresour. Technol. 157: 240-246.

35. Lu XQ, Ni JL, Zhen GY, Kubota K, Li YY. 2018. Response of morphology and microbial community structure of granules to influent $\mathrm{COD} / \mathrm{SO}_{4}{ }^{2-}$ ratios in an upflow anaerobic sludge blanket (UASB) reactor treating starch wastewater. Bioresour. Technol. 256: 456-465.

36. Sundberg C, Al-Soud WA, Larsson M, Alm E, Yekta SS, Svensson BH, et al. 2013. 454 pyrosequencing analyses of bacterial and archaeal richness in 21 full-scale biogas digesters. FEMS Microbiol. Ecol. 85: 612-626.

37. Kharayat Y. 2012. Distillery wastewater: bioremediation approaches. J. Integr. Environ. Sci. 9: 69-91.

38. Schmidt JE, Ahring BK. 1994. Extracellular polymers in granular sludge from different upflow anaerobic sludge blanket (UASB) reactors. Appl. Microbiol. Biotechnol. 42: 457462.

39. Ding JN, Wang DZ. 2005. Influence of external circulation on sludge characteristics during start-up of internal circulation reactor. J. Cent. South. Univ. T. 12: 425-429.

40. Wang JD, Xu WJ, Yan JJ, Yu JM. 2014. Study on the flow characteristics and the wastewater treatment performance in modified internal circulation reactor. Chemosphere 117: 631637.

41. Ueki A, Akasaka H, Satoh A, Suzuki D, Ueki K. 2007. Prevotella paludivivens sp. nov., a novel strictly anaerobic, Gram-negative, hemicellulose-decomposing bacterium isolated from plant residue and rice roots in irrigated rice-field soil. Int. J. Syst. Evol. Microbiol. 57: 1803-1809. 